\title{
Frequency control using a complex effective reflectivity in laterally coupled semiconductor laser arrays
}

\author{
Giora Griffel, William K. Marshall, Ilan Gravé, and Amnon Yariv \\ California Institute of Technology, Pasadena, California 91125 \\ Rashit Nabiev \\ P. N. Lebedev Physical Institute, Moscow 117924, USSR
}

\begin{abstract}
Received December 11, 1990
Frequency selectivity of a novel type of multielement, multisection laterally coupled semiconductor laser array is studied using the round-trip method. It is found that such a structure should lead to a strong frequency selectivity owing to a periodic dependency of the threshold gain on the frequency. A gain-guided two-coupledcavity device was fabricated. The experimental results show excellent agreement with the theoretical prediction.
\end{abstract}

It has been shown that laterally coupled ${ }^{1-3}$ and vertically coupled ${ }^{4}$ arrays of semiconductor lasers possess a strong frequency selectivity, manifested by the ability to control the longitudinal-mode spectrum and to tune the lasing wavelength. Round-trip analysis has been utilized in the past ${ }^{1}$ to calculate the laser threshold gain and lasing frequencies. In this Letter we extend round-trip analysis to allow for multilongitudinal sections and varying mirror reflectivities. The analysis is applied specifically to the case of two laterally coupled elements, each having two longitudinal electrodes, a long electrode for providing the gain and a short electrode near one facet used to control the phase and/or the magnitude of the reflectivity for this channel. In this way we create a complex, frequency-dependent, and electrically controlled effective reflectivity for the entire structure. This effective reflectivity is the key feature of this device, which enables us to control its lasing spectrum and to select a desired longitudinalmode operation. We show that for proper selection of the structural parameters in such a device, a strong frequency selectivity is obtained.

In general, the steady-state condition for a selfreproducing mode field leads to

$$
\mathbf{T}_{\mathrm{rt}} \cdot \mathbf{U}=\mathbf{U} \text {, }
$$

where $\mathbf{U}$ is a vector representing the optical field in terms of the isolated channel mode amplitudes and $\mathbf{T}_{\mathbf{r t}}$ is the round-trip propagator matrix. This matrix is obtained by a chain multiplication of individual matrices, each of which describes propagation within a longitudinal section, boundary crossing of two such sections, reflectivity at the end facet, or a representation change from a supermode form of field vector to a channel mode one and vice versa. It turns out that for the general case of two laterally coupled channels, each having a different (complex) end reflectivity, such as shown schematically in Fig. 1, the result of carrying the above matrix multiplication is

$$
\mathbf{T}_{\mathrm{rt}}=\left(p_{1} p_{2}\right)^{2}\left[\begin{array}{ll}
r_{1}\left(r_{2} A^{2}+r_{4} C^{2}\right) & r_{1}\left(r_{2} A+r_{4} B\right) C \\
r_{3}\left(r_{2} A+r_{4} B\right) C & r_{3}\left(r_{2} C^{2}+r_{4} B^{2}\right)
\end{array}\right]
$$

where $A, B$, and $C$ are given by

$$
\begin{aligned}
A & \equiv \frac{p_{1}}{p_{2}} \exp \left(i \sigma_{1} L\right)+\frac{p_{2}}{p_{1}} \exp \left(i \sigma_{2} L\right), \\
B & \equiv \frac{p_{1}}{p_{2}} \exp \left(i \sigma_{2} L\right)+\frac{p_{2}}{p_{1}} \exp \left(i \sigma_{1} L\right), \\
C & \equiv \exp \left(i \sigma_{1} L\right)-\exp \left(i \sigma_{2} L\right) .
\end{aligned}
$$

$L$ is the length of the device and $\sigma_{1,2}$ are the (complex) propagation constants of the compound (super) modes. These latter quantities are given by

$$
\sigma_{1,2}=\bar{\gamma} \pm s,
$$

where $s \equiv\left(\Delta \gamma \pm k_{a b} k_{b a}\right)^{1 / 2}, \Delta \gamma \equiv\left(\gamma_{a}-\gamma_{b}\right) / 2$, and $\bar{\gamma} \equiv\left(\gamma_{a}+\gamma_{b}\right) / 2 ; \gamma_{a, \mathrm{~b}}$ and $k_{a b, b a}$ are the propagation constants and the coupling coefficients of the isolated waveguides, respectively. The parameters $p_{1,2}$ in Eq. (2) are defined as

$$
p_{1,2}^{2}=\left(1 \pm \frac{\Delta \gamma}{s}\right) / 2
$$

The condition $\operatorname{det}\left(\mathbf{T}_{\mathrm{rt}}-\mathbf{I}\right)=0$, which follows from Eq. (1), leads, using Eq. (2), to

$$
\begin{aligned}
\left(p_{1} p_{2}\right)^{2}\left[r_{1} r_{2} A^{2}\right. & \left.+r_{3} r_{4} B^{2}+\left(r_{1} r_{4}+r_{2} r_{3}\right) C^{2}\right] \\
& -r_{1} r_{2} r_{3} r_{4} \exp \left[i 2\left(\sigma_{1}+\sigma_{2}\right) L\right]=1 .
\end{aligned}
$$

We now analyze the device depicted in Fig. 2. Here each channel has two longitudinal electrodes, a long one, which is forward biased to provide gain, and a short end-section electrode for controlling the phase and the magnitude of the reflectivity at the channel facets. For this specific case $r_{1}=r_{4} \equiv r$, $r_{2} \equiv \epsilon_{1} r \exp \left(i \phi_{1}\right)$, and $r_{3} \equiv \epsilon_{2} r \exp \left(i \phi_{2}\right)$. If we assume identical pumping conditions for the two gain sections, we also have $\gamma_{1}=\gamma_{2} \equiv \gamma, \Delta \gamma=0$, and $k_{a b}=$ 


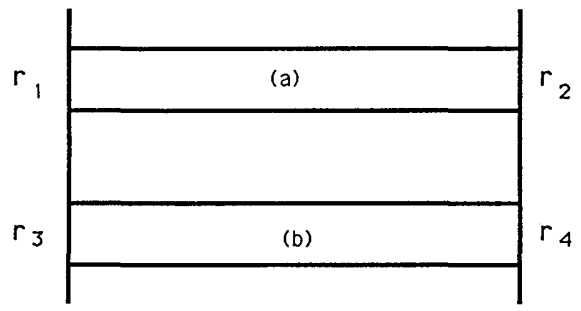

Fig. 1. Schematic of the laterally coupled two-channel [(a) and (b)] structure with different reflectivities at each channel facet.

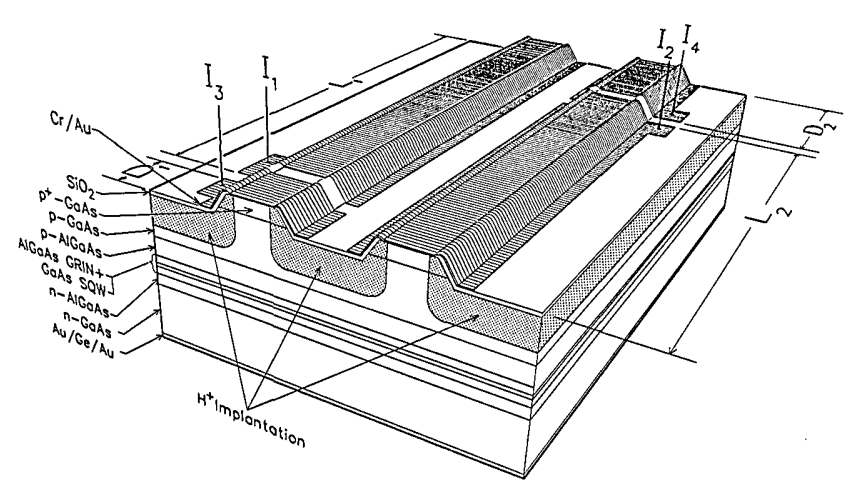

Fig. 2. Schematic of the proposed device. Each channel has two longitudinal electrodes, a gain (long) electrode and a phase/absorption (short) electrode.

$$
k_{b a} \equiv k \text {, hence } \sigma_{1,2}=\gamma \pm k, p_{1}=p_{2}=1 / \sqrt{2} \text {, and }
$$

$$
\begin{aligned}
A=B & =2 \exp (i \gamma L) \cos k L, \\
C & =2 i \exp (i \gamma L) \sin k L .
\end{aligned}
$$

Equation (6) becomes

$$
X^{2}-2 a X+1=0,
$$

where

$$
\begin{gathered}
X \equiv \sqrt{\epsilon_{1} \epsilon_{2}} r^{2} \exp (i 2 \gamma L) \exp (i \bar{\phi}), \\
a \equiv \frac{1}{2}\left\{\left[\sqrt{\frac{\epsilon_{1}}{\epsilon_{2}}} \exp (-i \Delta \phi)+\sqrt{\frac{\epsilon_{2}}{\epsilon_{1}}} \exp (i \Delta \phi)\right] \cos ^{2} k L\right. \\
\left.-\left[\frac{1}{\sqrt{\epsilon_{1} \epsilon_{2}}} \exp (-i \bar{\phi})+\sqrt{\epsilon_{1} \epsilon_{2}} \exp (i \bar{\phi})\right] \sin ^{2} k L\right\} .
\end{gathered}
$$

Here $\Delta \phi \equiv\left(\phi_{2}-\phi_{1}\right) / 2$ and $\bar{\phi} \equiv\left(\phi_{2}+\phi_{1}\right) / 2$, where $\phi_{1,2}=-2 \beta_{1,2} \Delta L_{1,2}$ is the phase contributed by each of the short end sections. $\beta_{1,2}$ is the propagation constant at each of the end-section regions. The solution to Eq. (8) is

$$
X=\exp \left( \pm i \cos ^{-1} a\right) .
$$

For the simple case of two laterally coupled indexguided lasers with no end sections, Eq. (11) reduces to $X=\exp ( \pm i 2 k L)$, i.e., the threshold condition is the same as that of the single channel except for a longitudinal Fabry-Perot mode splitting that is due to the appearance of lateral (super) modes. For the case of gain-guided channels, the parameter $a$ (and therefore $\varphi$ ) is a complex quantity, owing to $\epsilon_{1}, \epsilon_{2}$, or $k$ being a complex number. ${ }^{5}$ We have calculated the coupling coefficient for the case of two identical, equally pumped laterally coupled strip quantum-well lasers using the $\cosh ^{-2}$ lateral distribution for the modal gain. The result is shown in Fig. 3. It is seen that $k$ is a complex number whose phase is constantly increased with increasing gain $\gamma$. The magnitude of the coupling coefficient, although being proportional to the gain, tends to acquire a constant value owing to the increased confinement of the individual waveguide mode profiles.

Equation (10) indicates that the parameter $a$ has a periodic dependency on the frequency through $\phi_{1,2}$. Therefore, the threshold gain, obtained by equating the absolute values of each side of Eq. (11), is not constant but instead has a periodic frequency dependence. The threshold condition, as a function of the wavelength, for the case of a gain-guided structure with $\epsilon_{1}=\epsilon_{2}=1, L=600 \mu \mathrm{m}, D_{1}=45 \mu \mathrm{m}$, and $D_{2}=55 \mu \mathrm{m}$ was calculated. The results are shown in Fig. 4. The periodic behavior, with repetitive minima, gives rise to a frequency selectivity in the laser. Proper selection of the end-section lengths and the biasing current should therefore lead to single-longitudinal-mode operation.

In Fig. 5 we present experimental results of the configuration described in Fig. 2, utilizing a gainguided stripe laser structure as the channel elements. The stripe widths were $4 \mu \mathrm{m}$, and the center-to-center separation was $9 \mu \mathrm{m}$. The endsection electrodes were 215 and $195 \mu \mathrm{m}$, and the

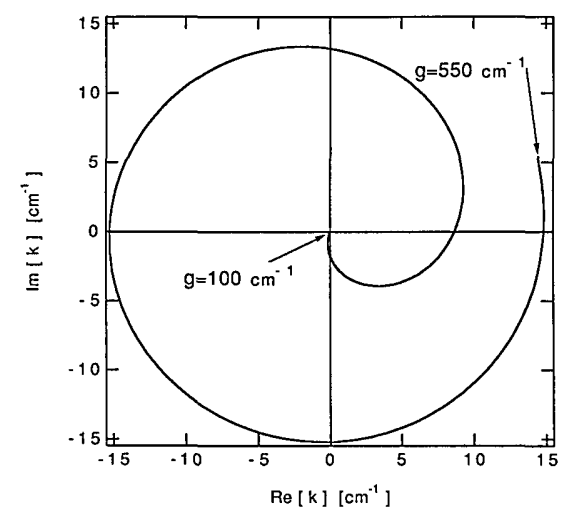

Fig. 3. Calculated coupling coefficient $k$ for the case of gain-guided coupled-cavity channels with identical gain in the two channels.

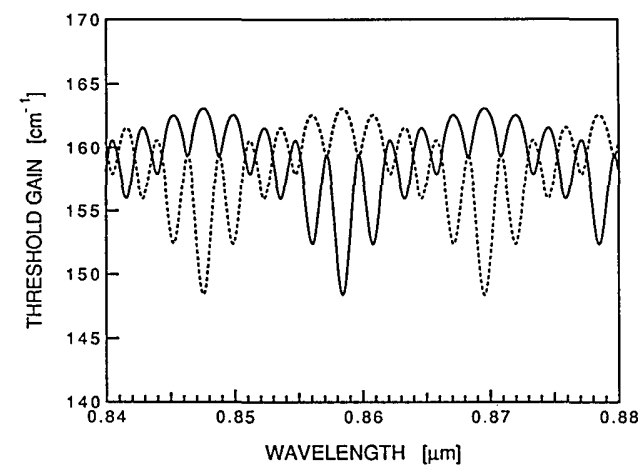

Fig. 4. Threshold gain versus frequency for the case of gain-guided channels with $\epsilon_{1}=\epsilon_{2}=1.0$ for the symmetric (solid curve) and antisymmetric (dashed curve) supermodes. 

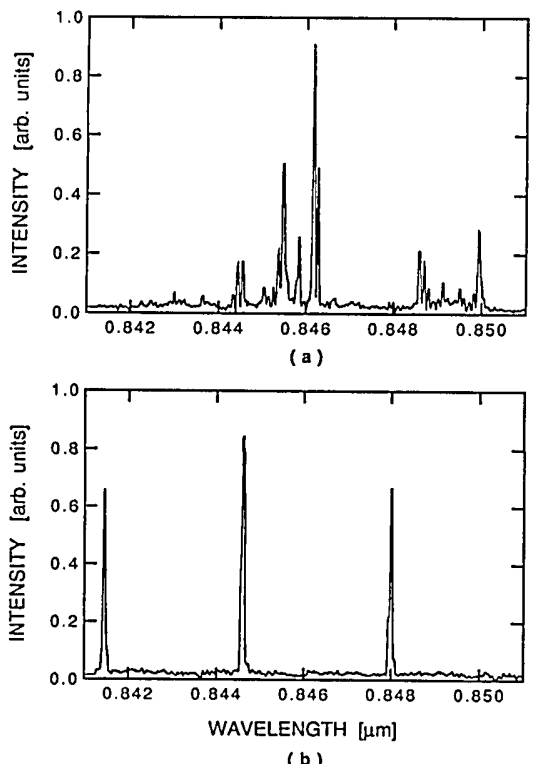

(b)

Fig. 5. Lasing spectrum of the proposed device under different operating conditions. (a) The end-section electrodes not biased (contacts opened), (b) the end-section electrodes shorted.

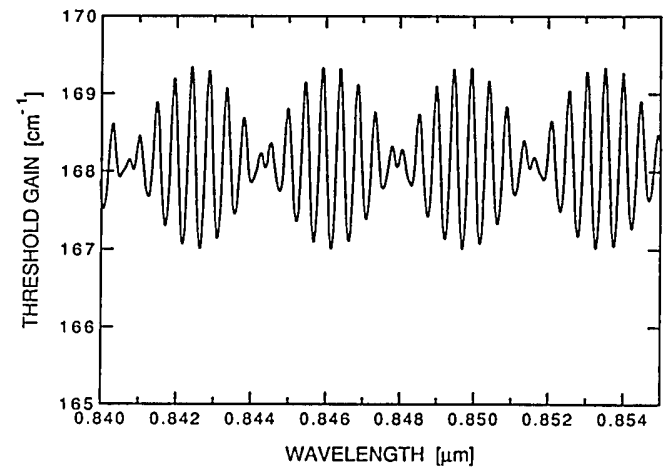

Fig. 6. Calculated threshold gain for the device of Fig. 5 with end sections shorted to ground $\left(\epsilon_{1,2}=0.03\right)$.

total length of the device was $600 \mu \mathrm{m}$. The laser spectra were measured for the cases of open-contact and shorted (0-V) end-section electrodes and are shown in Figs. 5(a) and 5(b), respectively. In the case of open contacts the end sections are bleached by a combined influence of light penetrating from the longitudinally coupled gain section and leakage current arriving from the adjacent electrode in the parallel channels. In that case the end sections will have little influence on the cavity loss, and as a result the device will function as an ordinary laterally coupled Fabry-Perot stripe laser, exhibiting a multimode spectrum as observed in the measured spectrum shown in Fig. 5(a). However, if the end- section electrodes are shorted instead, photoinduced carriers are swept away from the junction. Therefore these sections are not bleached, and they remain lossy. As a result their reflectivity will have a different magnitude and a different phase from those of the adjacent elements. The spectrum observed in this case is shown in Fig. 5(b). Here a strong frequency selectivity is observed, the whole spectrum shifts toward a shorter wavelength, and three isolated longitudinal modes are observed. The separation between these modes is equal to the equivalent of 27 longitudinal modes. A theoretical calculation of the threshold gain as a function of the frequency for the specific device parameters, with shorted end sections $\left(\epsilon_{1,2}=0.03\right)$, is shown in Fig. 6 . The calculation predicts minima separation of 28 longitudinal modes with $0.18-\mathrm{nm}$ separation between adjacent modes. The curve of Fig. 6 represents the gains that would be required for the structure to lase within the indicated wavelength range. In practice, the gain is clamped to the value at which the lowest threshold modes lase, i.e., $167 \mathrm{~cm}^{-1}$. The result is that only modes near the periodic minima can lase. The wavelength separation between these preferred modes is determined by the lengths of the sections in the device. The 28-longitudinal-mode separation agrees well with the observed spectrum of Fig. 5(b).

To conclude, we have analyzed multielectrode, laterally coupled-cavity semiconductor laser structures. A detailed round-trip analysis showed that such devices should exhibit strong frequency selectivity, which has been confirmed experimentally. Using this approach, one can construct single-longitudinal-mode devices with a fairly simple fabrication process.

Giora Griffel acknowledges the support of the Bantrell postdoctoral Fellowship at Caltech. This research was supported by the National Science Foundation, the Office of Naval Research, and the Army Research Office.

\section{References}

1. R. J. Lang, A. Yariv, and J. Salzman, IEEE J. Quantum Electron. QE-23, 395 (1987).

2. E. Kapon, J. Katz, S. Margalit, and A. Yariv, Appl. Phys. Lett. 44, 157 (1984).

3. Y. Suematsu, M. Yamada, and K. Hayashi, IEEE J. Quantum Electron. QE-11, 457 (1975).

4. S. Mukai, E. Kapon, J. Katz, C. Lindsey, Z. Rav-Noy, S. Margalit, and A. Yariv, Appl. Phys. Lett. 44, 478 (1984).

5. J. Katz, E. Kapon, C. Lindsey, S. Margalit, and A. Yariv, Appl. Opt. 23, 2231 (1984). 